\title{
Positive Solutions for Systems of Second-Order Difference Equations
}

\author{
Johnny Henderson ${ }^{1}$ and Rodica Luca ${ }^{2}$ \\ ${ }^{1}$ Department of Mathematics, Baylor University, Waco, TX 76798-7328, USA \\ ${ }^{2}$ Department of Mathematics, Gheorghe Asachi Technical University, 700506 Iasi, Romania \\ Correspondence should be addressed to Rodica Luca; rluca@math.tuiasi.ro
}

Received 17 March 2015; Accepted 30 August 2015

Academic Editor: Miguel Ángel López

Copyright (C) 2015 J. Henderson and R. Luca. This is an open access article distributed under the Creative Commons Attribution License, which permits unrestricted use, distribution, and reproduction in any medium, provided the original work is properly cited.

We study the existence and nonexistence of positive solutions of some systems of nonlinear second-order difference equations subject to multipoint boundary conditions which contain some positive constants.

\section{Introduction}

The mathematical modeling of many nonlinear problems from computer science, economics, mechanical engineering, control systems, biological neural networks, and others leads to the consideration of nonlinear difference equations (see $[1,2])$. In the last decades, many authors have investigated such problems by using various methods, such as fixed point theorems, the critical point theory, upper and lower solutions, the fixed point index theory, and the topological degree theory (see, e.g., [3-15]).

In this paper, we consider the system of nonlinear secondorder difference equations:

$$
\begin{array}{ll}
\Delta^{2} u_{n-1}+s_{n} f\left(v_{n}\right)=0, & n=\overline{1, N-1,} \\
\Delta^{2} v_{n-1}+t_{n} g\left(u_{n}\right)=0, & n=\overline{1, N-1,},
\end{array}
$$

with the multipoint boundary conditions

$$
\begin{aligned}
& u_{0}=\sum_{i=1}^{p} a_{i} u_{\xi_{i}}+a_{0}, \\
& u_{N}=\sum_{i=1}^{q} b_{i} u_{\eta_{i}},
\end{aligned}
$$

$$
\begin{aligned}
& v_{0}=\sum_{i=1}^{r} c_{i} v_{\zeta_{i}}, \\
& v_{N}=\sum_{i=1}^{l} d_{i} v_{\rho_{i}}+b_{0},
\end{aligned}
$$

where $N, p, q, r, l \in \mathbb{N}, N \geq 2, \Delta$ is the forward difference operator with stepsize $1, \Delta u_{n}=u_{n+1}-u_{n}, \Delta^{2} u_{n-1}=u_{n+1}-$ $2 u_{n}+u_{n-1}, n=\overline{k, m}$ means that $n=k, k+1, \ldots, m$ for $k, m \in \mathbb{N}$, $a_{i} \in \mathbb{R}$ for all $i=\overline{1, p}, b_{i} \in \mathbb{R}$ for all $i=\overline{1, q}, c_{i} \in \mathbb{R}$ for all $i=\overline{1, r}, d_{i} \in \mathbb{R}$ for all $i=\overline{1, l}, \xi_{i} \in \mathbb{N}$ for all $i=\overline{1, p}, \eta_{i} \in \mathbb{N}$ for all $i=\overline{1, q}, \zeta_{i} \in \mathbb{N}$ for all $i=\overline{1, r}, \rho_{i} \in \mathbb{N}$ for all $i=\overline{1, l}$, $1 \leq \xi_{1}<\cdots<\xi_{p} \leq N-1,1 \leq \eta_{1}<\cdots<\eta_{q} \leq N-1$, $1 \leq \zeta_{1}<\cdots<\zeta_{r} \leq N-1,1 \leq \rho_{1}<\cdots<\rho_{l} \leq N-1$, and $a_{0}$ and $b_{0}$ are positive constants.

Under some assumptions on the functions $f$ and $g$, we will prove the existence of positive solutions of problem $(S)$ (BC). By a positive solution of (S)-(BC) we mean a pair of sequences $\left(\left(u_{n}\right)_{n=0, N},\left(v_{n}\right)_{n=\overline{0, N}}\right)$ satisfying $(S)$ and $(B C)$ with $u_{n}, v_{n} \geq 0$ for all $n=\overline{0, N}$ and $u_{n}>0$ for all $n=\overline{0, N-1}$, $v_{n}>0$ for all $n=\overline{1, N}$. We will also give sufficient conditions for the nonexistence of positive solutions for this problem. System $(S)$ with the multipoint boundary conditions $\alpha u_{0}$ $\beta \Delta u_{0}=0, u_{N}=\sum_{i=1}^{m-2} a_{i} u_{\xi_{i}}+a_{0}, \gamma v_{0}-\delta \Delta v_{0}=0$, and 
$v_{N}=\sum_{i=1}^{p-2} b_{i} v_{\eta_{i}}+b_{0}\left(a_{0}, b_{0}>0\right)$ has been investigated in [16]. Some systems of difference equations with parameters, subject to multipoint boundary conditions, were studied in $[17,18]$, by using the Guo-Krasnosel'skii fixed point theorem. We also mention the paper [19], where the authors investigated the existence and multiplicity of positive solutions for the system $\Delta^{2} u_{n-1}+f\left(n, v_{n}\right)=0, \Delta^{2} v_{n-1}+g\left(n, u_{n}\right)=0$, $n=\overline{1, N-1}$, with the multipoint boundary conditions (BC) with $a_{0}=b_{0}=0$, by using some theorems from the fixed point index theory.

In Section 2, we present some auxiliary results which investigate a second-order difference equation subject to multipoint boundary conditions. In Section 3, we will prove our main results, and in Section 4, we will present an example which illustrates the obtained theorems. Our main existence result is based on the Schauder fixed point theorem which we present now.

Theorem 1. Let $X$ be a Banach space and $Y \subset X$ a nonempty, bounded, convex, and closed subset. If the operator $A: Y \rightarrow$ $Y$ is completely continuous (continuous and compact, i.e., mapping bounded sets into relatively compact sets), then A has at least one fixed point.

\section{Auxiliary Results}

In this section, we present some auxiliary results from [17] related to the following second-order difference equation with the multipoint boundary conditions:

$$
\begin{aligned}
\Delta^{2} u_{n-1}+y_{n} & =0, \quad n=\overline{1, N-1}, \\
u_{0} & =\sum_{i=1}^{p} a_{i} u_{\xi_{i}}, \\
u_{N} & =\sum_{i=1}^{q} b_{i} u_{\eta_{i}},
\end{aligned}
$$

where $N, p, q \in \mathbb{N}, N \geq 2, a_{i} \in \mathbb{R}$ for all $i=\overline{1, p}, b_{i} \in \mathbb{R}$ for all $i=\overline{1, q}, \xi_{i} \in \mathbb{N}$ for all $i=\overline{1, p}, \eta_{i} \in \mathbb{N}$ for all $i=\overline{1, q}$, $1 \leq \xi_{1}<\cdots<\xi_{p} \leq N-1$, and $1 \leq \eta_{1}<\cdots<\eta_{q} \leq N-1$.

Lemma 2 (see [17]). If $a_{i} \in \mathbb{R}$ for all $i=\overline{1, p}, b_{i} \in \mathbb{R}$ for all $i=\overline{1, q}, \xi_{i} \in \mathbb{N}$ for all $i=\overline{1, p}, \eta_{i} \in \mathbb{N}$ for all $i=\overline{1, q}$, $1 \leq \xi_{1}<\cdots<\xi_{p} \leq N-1,1 \leq \eta_{1}<\cdots<\eta_{q} \leq N-1$, $\Delta_{1}=\left(1-\sum_{i=1}^{q} b_{i}\right) \sum_{i=1}^{p} a_{i} \xi_{i}+\left(1-\sum_{i=1}^{p} a_{i}\right)\left(N-\sum_{i=1}^{q} b_{i} \eta_{i}\right) \neq 0$, and $y_{n} \in \mathbb{R}$ for all $n=\overline{1, N-1}$, then the solution of (1) is given by $u_{n}=\sum_{j=1}^{N-1} G_{1}(n, j) y_{j}$ for all $n=\overline{0, N}$, where Green's function $G_{1}$ is defined by

$$
\begin{aligned}
& G_{1}(n, j)=g_{0}(n, j) \\
& +\frac{1}{\Delta_{1}}\left[(N-n)\left(1-\sum_{k=1}^{q} b_{k}\right)+\sum_{i=1}^{q} b_{i}\left(N-\eta_{i}\right)\right] \\
& \cdot \sum_{i=1}^{p} a_{i} g_{0}\left(\xi_{i}, j\right)+\frac{1}{\Delta_{1}}\left[n\left(1-\sum_{k=1}^{p} a_{k}\right)+\sum_{i=1}^{p} a_{i} \xi_{i}\right] \\
& \cdot \sum_{i=1}^{q} b_{i} g_{0}\left(\eta_{i}, j\right), \quad n=\overline{0, N}, j=\overline{1, N-1},
\end{aligned}
$$

$$
g_{0}(n, j)=\frac{1}{N} \begin{cases}j(N-n), & 1 \leq j \leq n \leq N, \\ n(N-j), & 0 \leq n \leq j \leq N-1 .\end{cases}
$$

Lemma 3 (see [17]). If $a_{i} \geq 0$ for all $i=\overline{1, p}, \sum_{i=1}^{p} a_{i}<1$, $b_{i} \geq 0$ for all $i=\overline{1, q}, \sum_{i=1}^{q} b_{i}<1, \xi_{i} \in \mathbb{N}$ for all $i=\overline{1, p}$, $1 \leq \xi_{1}<\xi_{2}<\cdots<\xi_{p} \leq N-1, \eta_{i} \in \mathbb{N}$ for all $i=\overline{1, q}$, and $1 \leq \eta_{1}<\eta_{2}<\cdots<\eta_{q} \leq N-1$, then Green's function $G_{1}$ of problem (1) satisfies $G_{1}(n, j) \geq 0$ for all $n=\overline{0, N}, j=$ $\overline{1, N-1}$. Moreover, if $y_{n} \geq 0$ for all $n=\overline{1, N-1}$, then the unique solution $u_{n}, n=\overline{0, N}$, of problem (1) satisfies $u_{n} \geq 0$ for all $n=\overline{0, N}$.

Lemma 4 (see [17]). Assume that $a_{i} \geq 0$ for all $i=\overline{1, p}$, $\sum_{i=1}^{p} a_{i}<1, b_{i} \geq 0$ for all $i=\overline{1, q}, \sum_{i=1}^{q} b_{i}<1, \xi_{i} \in \mathbb{N}$ for all $i=\overline{1, p}, 1 \leq \xi_{1}<\xi_{2}<\cdots<\xi_{p} \leq N-1, \eta_{i} \in \mathbb{N}$ for all $i=\overline{1, q}$, and $1 \leq \eta_{1}<\eta_{2}<\cdots<\eta_{q} \leq N-1$. Then Green's function $G_{1}$ of problem (1) satisfies the following inequalities:

(a) $G_{1}(n, j) \leq I_{1}(j), \forall n=\overline{0, N}, j=\overline{1, N-1}$, where

$$
\begin{aligned}
I_{1}(j)= & g_{0}(j, j)+\frac{1}{\Delta_{1}}\left(N-\sum_{i=1}^{q} b_{i} \eta_{i}\right) \sum_{i=1}^{p} a_{i} g_{0}\left(\xi_{i}, j\right) \\
& +\frac{1}{\Delta_{1}}\left(N-\sum_{i=1}^{p} a_{i}\left(N-\xi_{i}\right)\right) \sum_{i=1}^{q} b_{i} g_{0}\left(\eta_{i}, j\right) .
\end{aligned}
$$

(b) For every $c \in\{1, \ldots, \llbracket N / 2 \rrbracket\}$, one has

$$
\begin{aligned}
\min _{n=\overline{c, N-c}} G_{1}(n, j) \geq \gamma_{1} I_{1}(j) \geq & \gamma_{1} G_{1}\left(n^{\prime}, j\right), \\
& \forall n^{\prime}=\overline{0, N}, j=\overline{1, N-1},
\end{aligned}
$$

where

$$
\begin{aligned}
\gamma_{1} & =\min \left\{\frac{c}{N-1}, \frac{c\left(1-\sum_{k=1}^{q} b_{k}\right)+\sum_{i=1}^{q} b_{i}\left(N-\eta_{i}\right)}{N-\sum_{i=1}^{q} b_{i} \eta_{i}},\right. \\
& \left.\frac{c\left(1-\sum_{k=1}^{p} a_{k}\right)+\sum_{i=1}^{p} a_{i} \xi_{i}}{N-\sum_{i=1}^{p} a_{i}\left(N-\xi_{i}\right)}\right\}>0,
\end{aligned}
$$

and $\llbracket N / 2 \rrbracket$ is the largest integer not greater than $N / 2$.

Lemma 5 (see [17]). Assume that $a_{i} \geq 0$ for all $i=\overline{1, p}$, $\sum_{i=1}^{p} a_{i}<1, b_{i} \geq 0$ for all $i=\overline{1, q}, \sum_{i=1}^{q} b_{i}<1, \xi_{i} \in \mathbb{N}$ for all $i=\overline{1, p}, 1 \leq \xi_{1}<\xi_{2}<\cdots<\xi_{p} \leq N-1, \eta_{i} \in \mathbb{N}$ for all $i=\overline{1, q}$, $1 \leq \eta_{1}<\eta_{2}<\cdots<\eta_{q} \leq N-1, c \in\{1, \ldots, \llbracket N / 2 \rrbracket\}$, and $y_{n} \geq 0$ for all $n=\overline{1, N-1}$. Then the solution $u_{n}, n=\overline{0, N}$, of problem (1) satisfies the inequality $\min _{n=c, N-c} u_{n} \geq \gamma_{1} \max _{m=\overline{0, N}} u_{m}$. 
We can also formulate similar results as Lemmas 2-5 above for the discrete boundary value problem

$$
\begin{aligned}
\Delta^{2} v_{n-1}+h_{n} & =0, \quad n=\overline{1, N-1} \\
v_{0}= & \sum_{i=1}^{r} c_{i} v_{\zeta_{i}} \\
v_{N} & =\sum_{i=1}^{l} d_{i} v_{\rho_{i}}
\end{aligned}
$$

where $N, r, l \in \mathbb{N}, N \geq 2, c_{i} \in \mathbb{R}$ for all $i=\overline{1, r}, d_{i} \in \mathbb{R}$ for all $i=\overline{1, l}, \zeta_{i} \in \mathbb{N}$ for all $i=\overline{1, r}, \rho_{i} \in \mathbb{N}$ for all $i=\overline{1, l}$, $1 \leq \zeta_{1}<\cdots<\zeta_{r} \leq N-1,1 \leq \rho_{1}<\cdots<\rho_{l} \leq N-1$, and $h_{n} \in \mathbb{R}$ for all $n=\overline{1, N-1}$. We denote by $\Delta_{2}, \gamma_{2}, G_{2}$, and $I_{2}$ the corresponding constants and functions for problem (6) defined in a similar manner as $\Delta_{1}, \gamma_{1}, G_{1}$, and $I_{1}$, respectively.

\section{Main Results}

We present first the assumptions that we will use in the sequel:

(H1) $\xi_{i} \in \mathbb{N}$ for all $i=\overline{1, p}, 1 \leq \xi_{1}<\cdots<\xi_{p} \leq N-1, a_{i} \geq 0$ for all $i=\overline{1, p}, \sum_{i=1}^{p} a_{i}<1, \eta_{i} \in \mathbb{N}$ for all $i=\overline{1, q}$, $1 \leq \eta_{1}<\cdots<\eta_{q} \leq N-1, b_{i} \geq 0$ for all $i=\overline{1, q}$, $\sum_{i=1}^{q} b_{i}<1, \zeta_{i} \in \mathbb{N}$ for all $i=\overline{1, r}, 1 \leq \zeta_{1}<\cdots<\zeta_{r} \leq$ $N-1, c_{i} \geq 0$ for all $i=\overline{1, r}, \sum_{i=1}^{r} c_{i}<1, \rho_{i} \in \mathbb{N}$ for all $i=\overline{1, l}, 1 \leq \rho_{1}<\cdots<\rho_{l} \leq N-1$, and $d_{i} \geq 0$ for all $i=\overline{1, l}, \sum_{i=1}^{l} d_{i}<1$.

(H2) The constants $s_{n}, t_{n} \geq 0$ for all $n=\overline{1, N-1}$, and there exist $i_{0}, j_{0} \in\{1, \ldots, N-1\}$ such that $s_{i_{0}}>0, t_{j_{0}}>0$.

$(H 3) f, g:[0, \infty) \rightarrow[0, \infty)$ are continuous functions and there exists $c_{0}>0$ such that $f(u)<c_{0} / L$, $g(u)<c_{0} / L$ for all $u \in\left[0, c_{0}\right]$, where $L=$ $\max \left\{\sum_{i=1}^{N-1} s_{i} I_{1}(i), \sum_{i=1}^{N-1} t_{i} I_{2}(i)\right\}$ and $I_{1}, I_{2}$ are defined in Section 2.

$(H 4) f, g:[0, \infty) \rightarrow[0, \infty)$ are continuous functions and satisfy the conditions $\lim _{u \rightarrow \infty}(f(u) / u)=$ $\infty, \lim _{u \rightarrow \infty}(g(u) / u)=\infty$.

Our first theorem is the following existence result for problem $(S)-(\mathrm{BC})$.

Theorem 6. Assume that assumptions (H1)-(H3) hold. Then problem (S)-(BC) has at least one positive solution for $a_{0}>0$ and $b_{0}>0$ sufficiently small.

Proof. We consider the problems

$$
\begin{aligned}
\Delta^{2} h_{n-1} & =0, \\
n & =\overline{1, N-1}, \\
h_{0} & =\sum_{i=1}^{p} a_{i} h_{\xi_{i}}+1, \\
h_{N} & =\sum_{i=1}^{q} b_{i} h_{\eta_{i}},
\end{aligned}
$$

$$
\begin{aligned}
\Delta^{2} k_{n-1} & =0, \\
n & =\overline{1, N-1}, \\
k_{0} & =\sum_{i=1}^{r} c_{i} k_{\zeta_{i}}, \\
k_{N} & =\sum_{i=1}^{l} d_{i} k_{\rho_{i}}+1 .
\end{aligned}
$$

Problems (7) and (8) have the solutions

$$
\begin{aligned}
& h_{n}=\frac{1}{\Delta_{1}}\left[-n\left(1-\sum_{i=1}^{q} b_{i}\right)+\left(N-\sum_{i=1}^{q} b_{i} \eta_{i}\right)\right], \\
& n=\overline{0, N}, \\
& k_{n}=\frac{1}{\Delta_{2}}\left[n\left(1-\sum_{i=1}^{r} c_{i}\right)+\sum_{i=1}^{r} c_{i} \zeta_{i}\right], \quad n=\overline{0, N},
\end{aligned}
$$

respectively, where $\Delta_{1}$ and $\Delta_{2}$ are defined in Section 2. By assumption $(H 1)$ we obtain $h_{n}>0$ for all $n=\overline{0, N-1}$ and $k_{n}>0$ for all $n=\overline{1, N}$.

We define the sequences $\left(x_{n}\right)_{n=\overline{0, N}},\left(y_{n}\right)_{n=\overline{0, N}}$ by

$$
\begin{aligned}
& x_{n}=u_{n}-a_{0} h_{n}, \\
& y_{n}=v_{n}-b_{0} k_{n},
\end{aligned}
$$

$$
n=\overline{0, N}
$$

where $\left(\left(u_{n}\right)_{n=\overline{0, N}},\left(v_{n}\right)_{n=\overline{0, N}}\right)$ is a solution of $(S)$-(BC). Then $(S)-(B C)$ can be equivalently written as

$$
\begin{array}{ll}
\Delta^{2} x_{n-1}+s_{n} f\left(y_{n}+b_{0} k_{n}\right)=0, & n=\overline{1, N-1}, \\
\Delta^{2} y_{n-1}+t_{n} g\left(x_{n}+a_{0} h_{n}\right)=0, & n=\overline{1, N-1,},
\end{array}
$$

with the boundary conditions

$$
\begin{aligned}
& x_{0}=\sum_{i=1}^{r} a_{i} x_{\xi_{i}}, \\
& x_{N}=\sum_{i=1}^{q} b_{i} x_{\eta_{i}}, \\
& y_{0}=\sum_{i=1}^{r} c_{i} y_{\zeta_{i}}, \\
& y_{N}=\sum_{i=1}^{l} d_{i} y_{\rho_{i}} .
\end{aligned}
$$


Using Green's functions $G_{1}$ and $G_{2}$ from Section 2, a pair $\left(\left(x_{n}\right)_{n=\overline{0, N}},\left(y_{n}\right)_{n=\overline{0, N}}\right)$ is a solution of problem (11)-(12) if and only if it is a solution for the problem

$$
\begin{aligned}
& x_{n}=\sum_{i=1}^{N-1} G_{1}(n, i) \\
& \cdot s_{i} f\left(\sum_{j=1}^{N-1} G_{2}(i, j) t_{j} g\left(x_{j}+a_{0} h_{j}\right)+b_{0} k_{i}\right), \\
& \quad n=\overline{0, N}, \\
& y_{n}=\sum_{i=1}^{N-1} G_{2}(n, i) t_{i} g\left(x_{i}+a_{0} h_{i}\right), \quad n=\overline{0, N},
\end{aligned}
$$

where $\left(h_{n}\right)_{n=\overline{0, N}},\left(k_{n}\right)_{n=\overline{0, N}}$ are given in (9).

We consider the Banach space $X=\mathbb{R}^{N+1}$ with the norm $\|u\|=\max _{n=\overline{0, N}}\left|u_{n}\right|, u=\left(u_{n}\right)_{n=\overline{0, N}}$, and we define the set $M=$ $\left\{\left(x_{n}\right)_{n=\overline{0, N}}, 0 \leq x_{n} \leq c_{0}, \forall n=\overline{0, N}\right\} \subset X$.

We also define the operator $\mathscr{E}: M \rightarrow X$ by

$$
\begin{array}{r}
\mathscr{E}(x)=\left(\sum_{i=1}^{N-1} G_{1}(n, i)\right. \\
\left.\cdot s_{i} f\left(\sum_{j=1}^{N-1} G_{2}(i, j) t_{j} g\left(x_{j}+a_{0} h_{j}\right)+b_{0} k_{i}\right)\right)_{n=\overline{0, N}}, \\
x=\left(x_{n}\right)_{n=\overline{0, N}} \in M .
\end{array}
$$

For sufficiently small $a_{0}>0$ and $b_{0}>0$, by (H3), we deduce

$$
\begin{aligned}
& f\left(y_{n}+b_{0} k_{n}\right) \leq \frac{c_{0}}{L}, \\
& g\left(x_{n}+a_{0} h_{n}\right) \leq \frac{c_{0}}{L},
\end{aligned}
$$

$$
\forall n=\overline{0, N}, \forall\left(x_{n}\right)_{n},\left(y_{n}\right)_{n} \in M .
$$

Then, by using Lemma 3 , we obtain $\mathscr{E}(x)_{n} \geq 0$ for all $n=$ $\overline{0, N}$ and $x=\left(x_{n}\right)_{n=\overline{0, N}} \in M$. By Lemma 4 , for all $x \in M$, we have

$$
\begin{aligned}
& \sum_{j=1}^{N-1} G_{2}(i, j) t_{j} g\left(x_{j}+a_{0} h_{j}\right) \\
& \leq \sum_{j=1}^{N-1} I_{2}(j) t_{j} g\left(x_{j}+a_{0} h_{j}\right) \leq \frac{c_{0}}{L} \sum_{j=1}^{N-1} t_{j} I_{2}(j) \leq c_{0}, \\
& \qquad i=\overline{1, N-1}, \\
& \mathscr{E}(x)_{n} \\
& \leq \sum_{i=1}^{N-1} I_{1}(i) s_{i} f\left(\sum_{j=1}^{N-1} G_{2}(i, j) t_{j} g\left(x_{j}+a_{0} h_{j}\right)+b_{0} k_{i}\right) \\
& \leq \frac{c_{0}}{L} \sum_{i=1}^{N-1} s_{i} I_{1}(i) \leq c_{0}, \quad \forall n=\overline{0, N} .
\end{aligned}
$$

Therefore $\mathscr{E}(M) \subset M$.
Using standard arguments, we deduce that $\mathscr{E}$ is completely continuous. By Theorem 1 , we conclude that $\mathscr{E}$ has a fixed point $x=\left(x_{n}\right)_{n=\overline{0, N}} \in M$. This element together with $y=\left(y_{n}\right)_{n=\overline{0, N}}$ given by (13) represents a solution for (11)-(12). This shows that our problem (S)-(BC) has a positive solution $\left(\left(u_{n}\right)_{n=\overline{0, N}},\left(v_{n}\right)_{n=\overline{0, N}}\right)$ with $u_{n}=x_{n}+a_{0} h_{n}, v_{n}=y_{n}+b_{0} k_{n}, n=$ $\overline{0, N},\left(u_{n}>0\right.$ for all $n=\overline{0, N-1}$ and $v_{n}>0$ for all $\left.n=\overline{1, N}\right)$ for sufficiently small $a_{0}>0$ and $b_{0}>0$.

In what follows, we present sufficient conditions for the nonexistence of positive solutions of $(S)-(B C)$.

Theorem 7. Assume that assumptions (H1), (H2), and (H4) hold. Then problem (S)-(BC) has no positive solution for $a_{0}$ and $b_{0}$ sufficiently large.

Proof. We suppose that $\left(\left(u_{n}\right)_{n=\overline{0, N}},\left(v_{n}\right)_{n=\overline{0, N}}\right)$ is a positive solution of (S)-(BC). Then $\left(\left(x_{n}\right)_{n=\overline{0, N}},\left(y_{n}\right)_{n=\overline{0, N}}\right)$ with $x_{n}=$ $u_{n}-a_{0} h_{n}, y_{n}=v_{n}-b_{0} k_{n}, n=\overline{0, N}$, is a solution for (11)-(12), where $\left(h_{n}\right)_{n=\overline{0, N}}$ and $\left(k_{n}\right)_{n=\overline{0, N}}$ are the solutions of problems (7) and (8), respectively (given by (9)). By (H2) there exists $c \in\{1,2, \ldots, \llbracket N / 2 \rrbracket\}$ such that $i_{0}, j_{0} \in\{c, \ldots, N-c\}$ and then $\sum_{i=c}^{N-c} s_{i} I_{1}(i)>0$ and $\sum_{i=c}^{N-c} t_{i} I_{2}(i)>0$. By using Lemma 3 we have $x_{n} \geq 0, y_{n} \geq 0$ for all $n=\overline{0, N}$, and by Lemma 5 , we obtain $\min _{n=\bar{c}, N-c} x_{n} \geq \gamma_{1}\|x\|$ and $\min _{n=\bar{c}, N-c} y_{n} \geq \gamma_{2}\|y\|$, where $\gamma_{1}$ and $\gamma_{2}$ are defined in Section 2.

Using now (9), we deduce that

$$
\begin{aligned}
& \min _{n=\overline{c, N-c}} h_{n}=h_{N-c}=\frac{h_{N-c}}{h_{0}}\|h\|, \\
& \min _{n=\overline{c, N-c}} k_{n}=k_{c}=\frac{k_{c}}{k_{N}}\|k\| .
\end{aligned}
$$

Therefore, we obtain

$$
\begin{aligned}
\min _{n=\overline{c, N-c}}\left(x_{n}+a_{0} h_{n}\right) & \geq \gamma_{1}\|x\|+\frac{a_{0} h_{N-c}}{h_{0}}\|h\| \\
& \geq r_{1}\left(\|x\|+a_{0}\|h\|\right) \\
& \geq r_{1}\left\|x+a_{0} h\right\|, \\
\min _{n=\overline{c, N-c}}\left(y_{n}+b_{0} k_{n}\right) & \geq \gamma_{2}\|y\|+\frac{b_{0} k_{c}}{k_{N}}\|k\| \\
& \geq r_{2}\left(\|y\|+b_{0}\|k\|\right) \geq r_{2}\left\|y+b_{0} k\right\|,
\end{aligned}
$$

where $r_{1}=\min \left\{\gamma_{1}, h_{N-c} / h_{0}\right\}$ and $r_{2}=\min \left\{\gamma_{2}, k_{c} / k_{N}\right\}$.

We now consider

$$
R=\left(\min \left\{\gamma_{2} r_{1} \sum_{i=c}^{N-c} t_{i} I_{2}(i), \gamma_{1} r_{2} \sum_{i=c}^{N-c} s_{i} I_{1}(i)\right\}\right)^{-1}>0
$$

By using (H4), for $R$ defined above, we conclude that there exists $M_{0}>0$ such that $f(u)>2 R u, g(u)>2 R u$ for 
all $u \geq M_{0}$. We consider $a_{0}>0$ and $b_{0}>0$ sufficiently large such that

$$
\begin{gathered}
\min _{n=\overline{c, N-c}}\left(x_{n}+a_{0} h_{n}\right) \geq M_{0}, \\
\min _{n=\overline{c, N-c}}\left(y_{n}+b_{0} k_{n}\right) \geq M_{0} .
\end{gathered}
$$

By (H2), (11), (12), and the above inequalities, we deduce that $\|x\|>0$ and $\|y\|>0$.

Now, by using Lemma 4 and the above considerations, we have

$$
\begin{aligned}
y_{c} & =\sum_{i=1}^{N-1} G_{2}(c, i) t_{i} g\left(x_{i}+a_{0} h_{i}\right) \\
& \geq \gamma_{2} \sum_{i=1}^{N-1} I_{2}(i) t_{i} g\left(x_{i}+a_{0} h_{i}\right) \\
& \geq \gamma_{2} \sum_{i=c}^{N-c} I_{2}(i) t_{i} g\left(x_{i}+a_{0} h_{i}\right) \\
& \geq 2 R \gamma_{2} \sum_{i=c}^{N-c} I_{2}(i) t_{i}\left(x_{i}+a_{0} h_{i}\right) \\
& \geq 2 R \gamma_{2} \sum_{i=c}^{N-c} I_{2}(i) t_{i} \min _{j=c, N-c}\left\{x_{j}+a_{0} h_{j}\right\} \\
& \geq 2 R \gamma_{2} r_{1} \sum_{i=c}^{N-c} I_{2}(i) t_{i}\left\|x+a_{0} h\right\| \geq 2\left\|x+a_{0} h\right\| \\
& \geq 2\|x\| .
\end{aligned}
$$

Therefore, we obtain

$$
\|x\| \leq \frac{y_{c}}{2} \leq \frac{\|y\|}{2} .
$$

In a similar manner, we deduce

$$
\begin{aligned}
x_{c} & =\sum_{i=1}^{N-1} G_{1}(c, i) s_{i} f\left(y_{i}+b_{0} k_{i}\right) \\
& \geq \gamma_{1} \sum_{i=1}^{N-1} I_{1}(i) s_{i} f\left(y_{i}+b_{0} k_{i}\right) \\
& \geq \gamma_{1} \sum_{i=c}^{N-c} I_{1}(i) s_{i} f\left(y_{i}+b_{0} k_{i}\right) \\
& \geq 2 R \gamma_{1} \sum_{i=c}^{N-c} I_{1}(i) s_{i}\left(y_{i}+b_{0} k_{i}\right) \\
& \geq 2 R \gamma_{1} \sum_{i=c}^{N-c} I_{1}(i) s_{i} \min _{j=c, N-c}\left\{y_{j}+b_{0} k_{j}\right\} \\
& \geq 2 R \gamma_{1} r_{2} \sum_{i=c}^{N-c} I_{1}(i) s_{i}\left\|y+b_{0} k\right\| \geq 2\left\|y+b_{0} k\right\| \\
& \geq 2\|y\| .
\end{aligned}
$$

So, we obtain

$$
\|y\| \leq \frac{x_{c}}{2} \leq \frac{\|x\|}{2}
$$

By (22) and (24), we obtain $\|x\| \leq\|y\| / 2 \leq\|x\| / 4$, which is a contradiction, because $\|x\|>0$. Then, for $a_{0}$ and $b_{0}$ sufficiently large, problem $(S)-(\mathrm{BC})$ has no positive solution.

Similar results as Theorems 6 and 7 can be obtained if instead of boundary conditions (BC) we have

$$
\begin{aligned}
& u_{0}=\sum_{i=1}^{p} a_{i} u_{\xi_{i}}, \\
& u_{N}=\sum_{i=1}^{q} b_{i} u_{\eta_{i}}+a_{0}, \\
& v_{0}=\sum_{i=1}^{r} c_{i} v_{\zeta_{i}}+b_{0}, \\
& v_{N}=\sum_{i=1}^{l} d_{i} v_{\rho_{i}}
\end{aligned}
$$

or

$$
\begin{aligned}
& u_{0}=\sum_{i=1}^{p} a_{i} u_{\xi_{i}}+a_{0}, \\
& u_{N}=\sum_{i=1}^{q} b_{i} u_{\eta_{i}}, \\
& v_{0}=\sum_{i=1}^{r} c_{i} v_{\zeta_{i}}+b_{0}, \\
& v_{N}=\sum_{i=1}^{l} d_{i} v_{\rho_{i}}
\end{aligned}
$$

or

$$
\begin{aligned}
& u_{0}=\sum_{i=1}^{p} a_{i} u_{\xi_{i}}, \\
& u_{N}=\sum_{i=1}^{q} b_{i} u_{\eta_{i}}+a_{0}, \\
& v_{0}=\sum_{i=1}^{r} c_{i} v_{\zeta_{i}}, \\
& v_{N}=\sum_{i=1}^{l} d_{i} v_{\rho_{i}}+b_{0},
\end{aligned}
$$

where $a_{0}$ and $b_{0}$ are positive constants. 
For problem $(S)-\left(\mathrm{BC}_{1}\right)$, instead of sequences $\left(h_{n}\right)_{n=\overline{0, N}}$ and $\left(k_{n}\right)_{n=\overline{0, N}}$ from the proof of Theorem 6 , the solutions of problems

$$
\begin{aligned}
\Delta^{2} \widetilde{h}_{n-1} & =0, \quad n=\overline{1, N-1}, \\
\widetilde{h}_{0} & =\sum_{i=1}^{p} a_{i} \widetilde{h}_{\xi_{i}}, \\
\widetilde{h}_{N} & =\sum_{i=1}^{q} b_{i} \widetilde{h}_{\eta_{i}}+1, \\
\Delta^{2} \widetilde{k}_{n-1} & =0, \quad n=\overline{1, N-1}, \\
\widetilde{k}_{0} & =\sum_{i=1}^{r} c_{i} \widetilde{k}_{\zeta_{i}}+1, \\
\widetilde{k}_{N} & =\sum_{i=1}^{l} d_{i} \widetilde{k}_{\rho_{i}}
\end{aligned}
$$

are

$$
\begin{array}{r}
\tilde{h}_{n}=\frac{1}{\Delta_{1}}\left[n\left(1-\sum_{i=1}^{p} a_{i}\right)+\sum_{i=1}^{p} a_{i} \xi_{i}\right], \quad n=\overline{0, N}, \\
\tilde{k}_{n}=\frac{1}{\Delta_{2}}\left[-n\left(1-\sum_{i=1}^{l} d_{i}\right)+\left(N-\sum_{i=1}^{l} d_{i} \rho_{i}\right)\right], \\
n=\overline{0, N},
\end{array}
$$

respectively. By assumption $(H 1)$ we obtain $\widetilde{h}_{n}>0$, for all $n=\overline{1, N}$, and $\widetilde{k}_{n}>0$ for all $n=\overline{0, N-1}$.

For problem $(S)-\left(\mathrm{BC}_{2}\right)$, instead of sequences $\left(h_{n}\right)_{n=\overline{0, N}}$ and $\left(k_{n}\right)_{k=\overline{0, N}}$ from Theorem 6 , the solutions of problems (7) and (26) are $\left(h_{n}\right)_{n=\overline{0, N}}$ and $\left(\widetilde{k}_{n}\right)_{n=\overline{0, N}}$, respectively, which satisfy $h_{n}>0$, for all $n=\overline{0, N-1}$, and $\widetilde{k}_{n}>0$ for all $n=\overline{0, N-1}$. For problem $(S)-\left(\mathrm{BC}_{3}\right)$, instead of sequences $\left(h_{n}\right)_{n=\overline{0, N}}$ and $\left(k_{n}\right)_{k=\overline{0, N}}$ from Theorem 6 , the solutions of problems (25) and (8) are $\left(\widetilde{h}_{n}\right)_{n=\overline{0, N}}$ and $\left(k_{n}\right)_{n=\overline{0, N}}$, respectively, which satisfy $\widetilde{h}_{n}>$ 0 , for all $n=\overline{1, N}$, and $k_{n}>0$ for all $n=\overline{1, N}$.

Therefore we also obtain the following results.

Theorem 8. Assume that assumptions (H1)-(H3) hold. Then problem $(S)-\left(\mathrm{BC}_{1}\right)$ has at least one positive solution $\left(u_{n}>0\right.$, for all $n=\overline{1, N}$, and $v_{n}>0$ for all $n=\overline{0, N-1}$ ) for $a_{0}>0$ and $b_{0}>0$ sufficiently small.

Theorem 9. Assume that assumptions (H1), (H2), and (H4) hold. Then problem $(S)-\left(\mathrm{BC}_{1}\right)$ has no positive solution $\left(u_{n}>0\right.$, for all $n=\overline{1, N}$, and $v_{n}>0$ for all $n=\overline{0, N-1}$ ) for $a_{0}$ and $b_{0}$ sufficiently large.
Theorem 10. Assume that assumptions (H1)-(H3) hold. Then problem $(S)-\left(\mathrm{BC}_{2}\right)$ has at least one positive solution $\left(u_{n}>0\right.$, for all $n=\overline{0, N-1}$, and $v_{n}>0$ for all $n=\overline{0, N-1}$ ) for $a_{0}>0$ and $b_{0}>0$ sufficiently small.

Theorem 11. Assume that assumptions (H1), (H2), and (H4) hold. Then problem $(S)-\left(\mathrm{BC}_{2}\right)$ has no positive solution $\left(u_{n}>0\right.$, for all $n=\overline{0, N-1}$, and $v_{n}>0$ for all $n=\overline{0, N-1}$ ) for $a_{0}$ and $b_{0}$ sufficiently large.

Theorem 12. Assume that assumptions (H1)-(H3) hold. Then problem $(S)-\left(\mathrm{BC}_{3}\right)$ has at least one positive solution $\left(u_{n}>0\right.$, for all $n=\overline{1, N}$, and $v_{n}>0$ for all $\left.n=\overline{1, N}\right)$ for $a_{0}>0$ and $b_{0}>0$ sufficiently small.

Theorem 13. Assume that assumptions (H1), (H2), and (H4) hold. Then problem $(S)-\left(\mathrm{BC}_{3}\right)$ has no positive solution $\left(u_{n}>0\right.$, for all $n=\overline{1, N}$, and $v_{n}>0$ for all $n=\overline{1, N}$ ) for $a_{0}$ and $b_{0}$ sufficiently large.

\section{An Example}

We consider $N=20, s_{n}=\widehat{c} /(n+1), t_{n}=\widehat{d} / n$ for all $n=\overline{1,19}$, $\widehat{c}>0, \widehat{d}>0, p=2, q=3, r=1, l=2, a_{1}=1 / 2, a_{2}=1 / 3$, $\xi_{1}=4, \xi_{2}=16, b_{1}=1 / 3, b_{2}=1 / 4, b_{3}=1 / 5, \eta_{1}=5, \eta_{2}=10$, $\eta_{3}=15, c_{1}=3 / 4, \zeta_{1}=10, d_{1}=1 / 3, d_{2}=1 / 5, \rho_{1}=3$, and $\rho_{2}=18$. We also consider the functions $f, g:[0, \infty) \rightarrow$ $[0, \infty), f(x)=\tilde{a} x^{\alpha_{1}} /(2 x+1)$, and $g(x)=\tilde{b} x^{\alpha_{2}} /(3 x+2)$, for all $x \in[0, \infty)$, with $\tilde{a}, \tilde{b}>0$ and $\alpha_{1}, \alpha_{2}>2$. We have $\lim _{x \rightarrow \infty} f(x) / x=\lim _{x \rightarrow \infty} g(x) / x=\infty$.

Therefore, we consider the system of second-order difference equations

$$
\begin{aligned}
\Delta^{2} u_{n-1}+\frac{\widehat{c} \widetilde{a} v_{n}^{\alpha_{1}}}{(n+1)\left(2 v_{n}+1\right)} & =0, \quad n=\overline{1,19}, \\
\Delta^{2} v_{n-1}+\frac{\widetilde{d} b u_{n}^{\alpha_{2}}}{n\left(3 u_{n}+2\right)} & =0, \quad n=\overline{1,19},
\end{aligned}
$$

with the multipoint boundary conditions

$$
\begin{aligned}
u_{0} & =\frac{1}{2} u_{4}+\frac{1}{3} u_{16}+a_{0}, \\
u_{20} & =\frac{1}{3} u_{5}+\frac{1}{4} u_{10}+\frac{1}{5} u_{15}, \\
v_{0} & =\frac{3}{4} v_{10}, \\
v_{20} & =\frac{1}{3} v_{3}+\frac{1}{5} v_{18}+b_{0},
\end{aligned}
$$

where $a_{0}$ and $b_{0}$ are positive constants. 
We have $\sum_{i=1}^{2} a_{i}=5 / 6<1, \sum_{i=1}^{3} b_{i}=47 / 60<1, \sum_{i=1}^{1} c_{i}=$ $3 / 4<1$, and $\sum_{i=1}^{2} d_{i}=8 / 15<1$. The functions $I_{1}$ and $I_{2}$ are given by

$$
\begin{gathered}
I_{1}(j)= \begin{cases}\frac{2565 j}{671}-\frac{j^{2}}{20}, & 1 \leq j \leq 4, \\
\frac{7820}{671}+\frac{70 j}{61}-\frac{j^{2}}{20}, & 5 \leq j \leq 9, \\
\frac{12620}{671}+\frac{290 j}{671}-\frac{j^{2}}{20}, & 10 \leq j \leq 15, \\
\frac{30700}{671}-\frac{864 j}{671}-\frac{j^{2}}{20}, & 16 \leq j \leq 19 .\end{cases} \\
I_{2}(j)= \begin{cases}\frac{145 j}{63}-\frac{j^{2}}{20}, & 1 \leq j \leq 2, \\
\frac{250}{147}+\frac{85 j}{49}-\frac{j^{2}}{20}, & 3 \leq j \leq 9, \\
\frac{2560}{147}+\frac{8 j}{49}-\frac{j^{2}}{20}, & 10 \leq j \leq 17, \\
\frac{3460}{147}-\frac{26 j}{147}-\frac{j^{2}}{20}, & 18 \leq j \leq 19 .\end{cases}
\end{gathered}
$$

Hence, we deduce that assumptions $(H 1),(H 2)$, and $(H 4)$ are satisfied. In addition, by using the above functions $I_{1}$ and $I_{2}$, we obtain $\widetilde{A}:=\sum_{i=1}^{19} I_{1}(i) /(i+1) \approx 30.1784002$, $\widetilde{B}:=\sum_{i=1}^{19} I_{2}(i) / i \approx 23.63831254$, and then $L=\max \{\widehat{c} \widetilde{A}, \hat{d} \widetilde{B}\}$. We choose $c_{0}=1$ and if we select $\widetilde{a}, \widetilde{b}$ satisfying the conditions $\widetilde{a}<3 / L=3 \min \{1 /(\widehat{c} \widetilde{A}), 1 /(\widehat{d} \widetilde{B})\}, \widetilde{b}<5 / L=$ $5 \min \{1 /(\widehat{c} \widetilde{A}), 1 /(\widehat{d} \widetilde{B})\}$, then we conclude that $f(x) \leq \widetilde{a} / 3<$ $1 / L, g(x) \leq \widetilde{b} / 5<1 / L$ for all $x \in[0,1]$. For example, if $\widehat{c}=1$, $\widehat{d}=2$, then for $\widetilde{a} \leq 0.063$ and $\widetilde{b} \leq 0.105$ the above conditions for $f$ and $g$ are satisfied. So, assumption (H3) is also satisfied. By Theorems 6 and 7 we deduce that problem $\left(S_{0}\right)-\left(\mathrm{BC}_{0}\right)$ has at least one positive solution (here $u_{n}>0$ and $v_{n}>0$ for all $n=\overline{0,20}$ ) for sufficiently small $a_{0}>0$ and $b_{0}>0$ and no positive solution for sufficiently large $a_{0}$ and $b_{0}$.

By the proofs of Theorems 6 and 7 we can find some intervals for $a_{0}$ and $b_{0}$ such that problem $\left(S_{0}\right)-\left(\mathrm{BC}_{0}\right)$ has at least one positive solution, or it has no positive solution. We consider $\widetilde{a}=0.063, \widetilde{b}=0.105, \widehat{c}=1, \widehat{d}=2, c_{0}=1$, $L=\max \{\widetilde{A}, 2 \widetilde{B}\}=2 \widetilde{B}$ (as above), $\alpha_{1}=3$, and $\alpha_{2}=4$. Then $\Delta_{1}=671 / 180, \Delta_{2}=147 / 20$, and the sequences $\left(h_{n}\right)_{n=\overline{0,20}}$ and $\left(k_{n}\right)_{n=0,20}$ from (9) are $h_{n}=(-39 n+2310) / 671$ and $k_{n}=(5 n+150) / 147$ for all $n=\overline{0,20}$. We also obtain $h_{\max }=h_{0}=210 / 61$ and $k_{\max }=k_{20}=250 / 147$. If we choose $b_{0} \leq\left(f^{-1}(1 / L)-1\right) / k_{\max }$ and $a_{0} \leq\left(g^{-1}(1 / L)-1\right) / h_{\max }$, then inequalities (15) are satisfied. Because $f^{-1}(1 / L) \approx 1.0031$ and $g^{-1}(1 / L) \approx 1.00213$, for $a_{0} \leq 6.17 \cdot 10^{-4}$ and $b_{0} \leq 18.21 \cdot 10^{-4}$ problem $\left(S_{0}\right)-\left(\mathrm{BC}_{0}\right)$ has at least one positive solution.

Now we choose $c=5$ (the constant from the beginning of the proof of Theorem 7), and then we obtain $\gamma_{1}=\gamma_{2}=5 / 19$, $h_{15}=1725 / 671, k_{5}=175 / 147, r_{1}=\min \left\{\gamma_{1}, h_{15} / h_{0}\right\}=5 / 19$, $r_{2}=\min \left\{\gamma_{2}, k_{5} / k_{20}\right\}=5 / 19, \sum_{i=5}^{15} t_{i} I_{2}(i) \approx 27.88321391$, $\sum_{i=5}^{15} s_{i} I_{1}(i) \approx 18.41083231$, and $R \approx 0.78432087$ (given by (19)). For $\widetilde{R}:=2 R+0.1$, the inequalities $f(x) / x \geq \widetilde{R}$ and $g(y) / y \geq \widetilde{R}$ are satisfied for $x \geq M_{0}^{\prime} \approx 53.468121231$ and $y \geq M_{0}^{\prime \prime} \approx 7.2166269$, respectively. We consider $M_{0}=\max \left\{M_{0}^{\prime}, M_{0}^{\prime \prime}\right\}=M_{0}^{\prime}$, and then for $a_{0} \geq M_{0} / h_{15}$ and $b_{0} \geq M_{0} / k_{5}$, inequalities (20) are satisfied. Therefore, if $a_{0} \geq 20.7984$ and $b_{0} \geq 44.9133$, problem $\left(S_{0}\right)-\left(\mathrm{BC}_{0}\right)$ has no positive solution.

\section{Conflict of Interests}

The authors declare that there is no conflict of interests regarding the publication of this paper.

\section{Acknowledgments}

The authors thank the referees for their valuable comments and suggestions. The work of R. Luca was supported by a grant of the Romanian National Authority for Scientific Research, CNCS-UEFISCDI, Project no. PN-II-ID-PCE2011-3-0557.

\section{References}

[1] W. G. Kelley and A. C. Peterson, Difference Equations. An Introduction with Applications, Academic Press, San Diego, Calif, USA, 2nd edition, 2001.

[2] V. Lakshmikantham and D. Trigiante, Theory of Difference Equations: Numerical Methods and Applications, vol. 181 of Mathematics is Science and Engineering, Academic Press, Boston, Mass, USA, 1988.

[3] G. A. Afrouzi and A. Hadjian, "Existence and multiplicity of solutions for a discrete nonlinear boundary value problem," Electronic Journal of Differential Equations, vol. 2014, no. 35, pp. 1-13, 2014.

[4] D. R. Anderson, "Solutions to second-order three-point problems on time scales," Journal of Difference Equations and Applications, vol. 8, no. 8, pp. 673-688, 2002.

[5] R. I. Avery, "Three positive solutions of a discrete second order conjugate problem," Panamerican Mathematical Journal, vol. 8, no. 2, pp. 79-96, 1998.

[6] W.-S. Cheung and J. Ren, "Positive solutions for discrete threepoint boundary value problems," The Australian Journal of Mathematical Analysis and Applications, vol. 1, pp. 1-7, 2004.

[7] C. S. Goodrich, "Existence of a positive solution to a nonlocal semipositone boundary value problem on a time scale," Commentationes Mathematicae Universitatis Carolinae, vol. 54, no. 4, pp. 509-525, 2013.

[8] J. R. Graef, L. Kong, and M. Wang, "Multiple solutions to a periodic boundary value problem for a nonlinear discrete fourth order equation," Advances in Dynamical Systems and Applications, vol. 8, no. 2, pp. 203-215, 2013.

[9] J. R. Graef, L. Kong, and M. Wang, "Existence of multiple solutions to a discrete fourth order periodic boundary value problem," Discrete and Continuous Dynamical Systems, supplement, pp. 291-299, 2013.

[10] A. Iannizzotto and S. A. Tersian, "Multiple homoclinic solutions for the discrete p-Laplacian via critical point theory," Journal of Mathematical Analysis and Applications, vol. 403, no. 1, pp. 173182, 2013.

[11] W. T. Li and H. R. Sun, "Positive solutions for second-order mpoint boundary value problems on times scales," Acta Mathematica Sinica, vol. 22, no. 6, pp. 1797-1804, 2006. 
[12] J. Rodriguez, "Nonlinear discrete Sturm-Liouville problems," Journal of Mathematical Analysis and Applications, vol. 308, no. 1, pp. 380-391, 2005.

[13] H.-R. Sun and W.-T. Li, "Positive solutions for nonlinear threepoint boundary value problems on time scales," Journal of Mathematical Analysis and Applications, vol. 299, no. 2, pp. 508$524,2004$.

[14] D.-B. Wang and W. Guan, "Three positive solutions of boundary value problems for p-Laplacian difference equations," Computers \& Mathematics with Applications, vol. 55, no. 9, pp. 19431949, 2008.

[15] L. Wang and X. Chen, "Positive solutions for discrete boundary value problems to one-dimensional p-Laplacian with delay," Journal of Applied Mathematics, vol. 2013, Article ID 157043, 8 pages, 2013.

[16] J. Henderson and R. Luca, "On a multi-point discrete boundary value problem," Journal of Difference Equations and Applications, vol. 19, no. 4, pp. 690-699, 2013.

[17] J. Henderson and R. Luca, "Existence of positive solutions for a system of second-order multi-point discrete boundary value problems," Journal of Difference Equations and Applications, vol. 19, no. 11, pp. 1889-1906, 2013.

[18] J. Henderson and R. Luca, "On a second-order nonlinear discrete multi-point eigenvalue problem," Journal of Difference Equations and Applications, vol. 20, no. 7, pp. 1005-1018, 2014.

[19] J. Henderson, R. Luca, and A. Tudorache, "Multiple positive solutions for a multi-point discrete boundary value problem," Communications, Faculty of Sciences, University of Ankara. Series A1: Mathematics and Statistics, vol. 63, no. 2, pp. 59-70, 2014. 


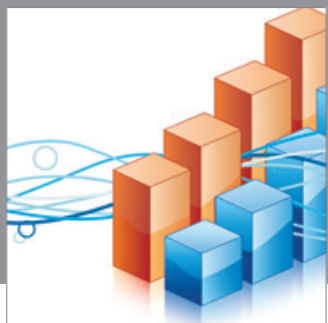

Advances in

Operations Research

mansans

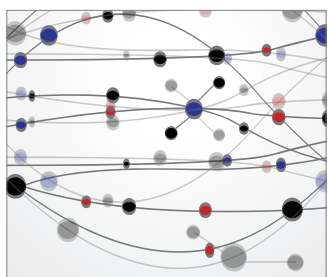

The Scientific World Journal
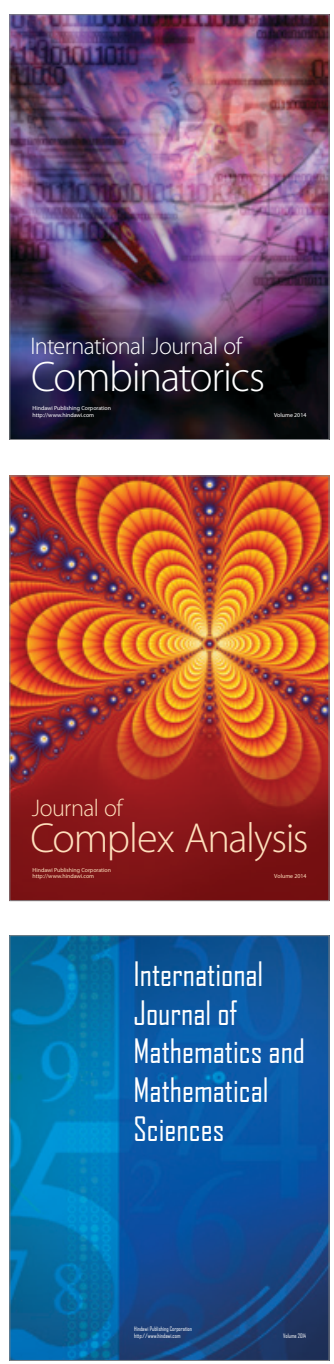
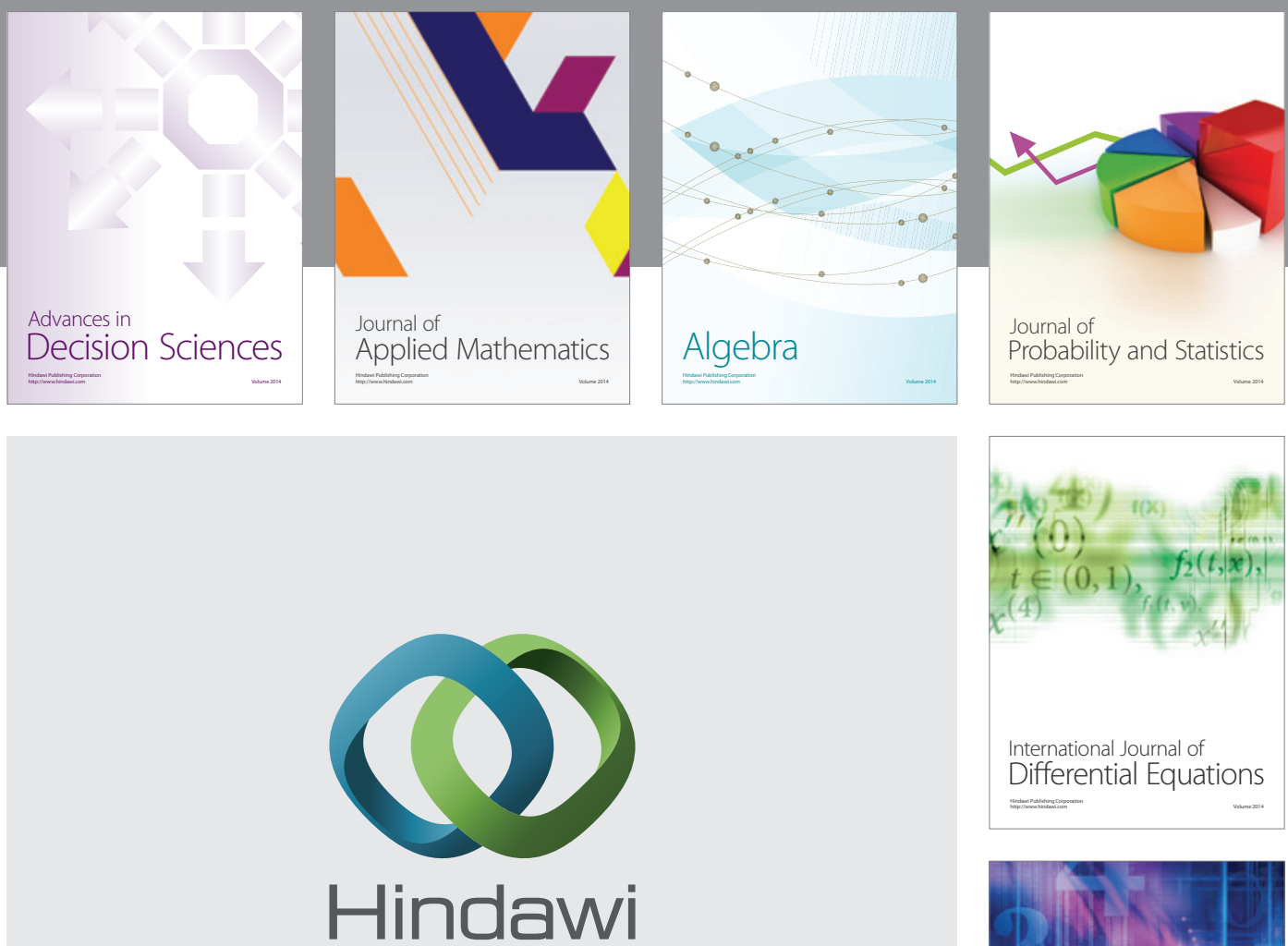

Submit your manuscripts at http://www.hindawi.com
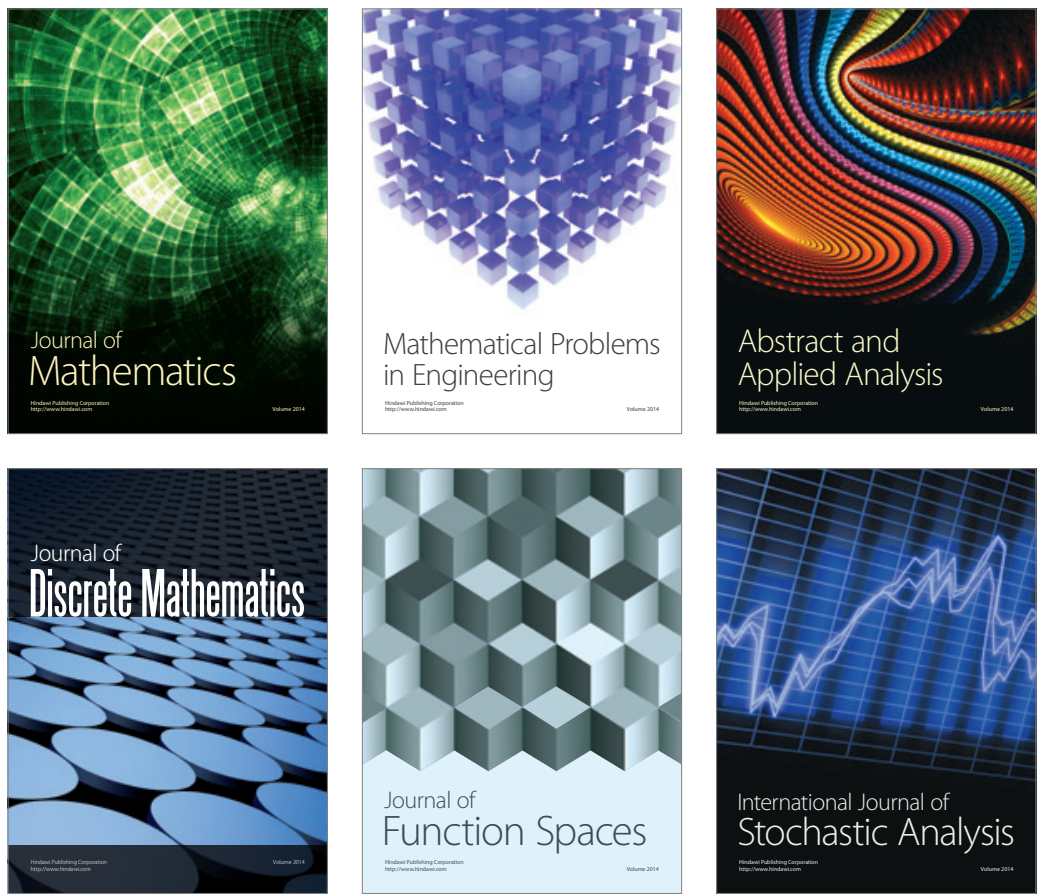

Journal of

Function Spaces

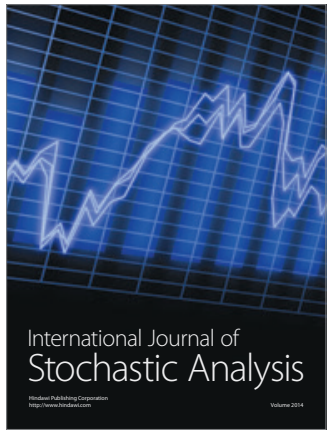

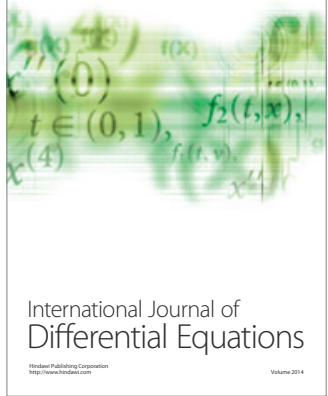
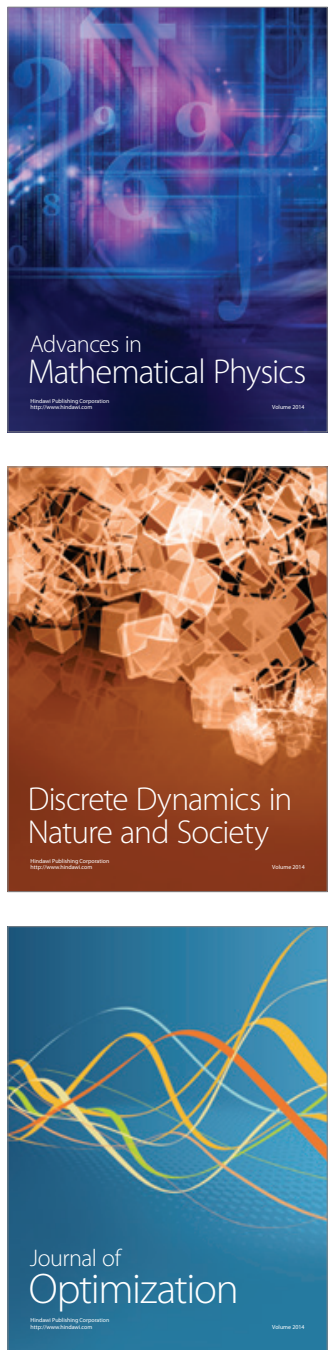